\title{
Polypes festonnés du côlon proximal : prenez votre temps !
}

\author{
Serrated polyps of the proximal colon: take your time!
}

\author{
G. Lesur \\ C Springer-Verlag France 2013
}

Contrairement aux autres localisations, il n'est pas prouvé que la coloscopie diminue la mortalité et l'incidence du cancer du côlon droit. Cette absence d'effet est probablement liée à plusieurs circonstances potentiellement associées, une préparation souvent moins bonne à droite, la reconnaissance à tort du bas-fond cæcal, alors qu'il s'agit de l'angle colique droit et aussi sans doute à ces désormais fameux polypes festonnés à haut risque évolutifs et souvent difficiles à repérer. Une passionnante étude hollandaise publiée dans le numéro d'avril de Gastrointestinal Endoscopy apporte sur ces polypes festonnés du côlon proximal un très intéressant éclairage [1].

Cette étude prospective a réuni les coloscopies d'un programme de dépistage réalisé dans deux centres universitaires d'Amsterdam. Entre juin 2009 et juillet 2010, 6000 sujets étaient invités à faire une coloscopie. Les sujets à risque moyen et élevé ou explorés dans les cinq ans précédant par coloscopie étaient exclus. Cinq endoscopistes expérimentés avec 6 à 35 ans de pratique de l'endoscopie digestive réalisèrent au total 1354 coloscopies. Cinquante et un pour cent des participants étaient des hommes, et il s'agissait de sujets d'âge moyen de 60 ans. Le score moyen d'Ottawa pour la préparation était de 5 et le temps moyen de retrait de l'endoscope de dix minutes. Mille six cent trentecinq polypes étaient détectés dont $43 \%$ d'adénomes et $42 \%$ de polypes festonnés. Le nombre moyen d'adénomes était de $0,52 \pm 1,08$ par patient. Le nombre moyen de polypes festonnés était de $0,51 \pm 1,16$ par patient, et $31 \%$ de ces polypes, détectés chez $12 \%$ des patients, étaient proximaux (définis ici comme situés au-delà de l'angle gauche). Le nombre moyen de polypes festonnés proximaux par patient était de 0,16 $\pm 0,48$ avec une taille moyenne de $4 \mathrm{~mm}$. Un ou plusieurs adénomes étaient détectés chez $29 \%$ des sujets avec une taille moyenne de $4 \mathrm{~mm}$. Un adénome avancé était détecté dans $9 \%$ des cas et un cancer dans $9 \%$ des cas.
Parmi ces endoscopistes expérimentés, les taux de détection de polypes festonnés variaient de 6 à $22 \%$ et celui des adénomes de 24 à $40 \%$. Quand étaient inclus l'âge, le sexe et la qualité de la préparation, les différences entre endoscopistes étaient significatives pour les polypes festonnés comme pour les adénomes $(p<0,001)$. Le temps de retrait de l'endoscope variait également de huit à seize minutes $(p<0,001)$. En analyse multivariée, la durée de l'examen au retrait de l'endoscope était le seul facteur indépendant associé à la découverte de polypes festonnés proximaux (OR : 1,12 ; [IC $95 \%=1,10-1,16])$. Il est intéressant de noter que l'endoscopiste qui détectait le plus d'adénomes était le quatrième en termes de détection de polypes festonnés, et que celui qui détectait le plus de polypes festonnés était le troisième en termes de détection d'adénomes. En analyse multivariée, un âge élevé et un temps de retrait long étaient également significativement associés à une meilleure détection des adénomes $(p<0,001)$. On remarquera aussi qu'une plus grande détection de polypes festonnés était significativement associée à une meilleure détection des adénomes, des adénomes avancés et des cancers.

Voltaire avec son sens de la formule disait : « il faut prendre le temps de faire court $»$. En détournant de manière intéressée cet esprit libre, on pourrait écrire : « en coloscopie, prenons le temps de faire long ». Cela est vrai pour tout le côlon, et tout particulièrement pour le côlon proximal.

\section{Référence}

1. de Wijkerslooth TR, Stoop EM, Bossuyt PM, Tytgat KM, Dees J, Mathus-Vliegen EM, et al. Differences in proximal serrated polyp detection among endoscopists are associated with variability in withdrawal time. Gastrointest Endosc 2013;77:617-23.
G. Lesur $(\bowtie)$

e-mail : gilles.lesur@apr.aphp.fr 